DESY 07-207

OUTP-0715P

$\mathrm{IPPP} / 07 / 93$

DCPT $/ 07 / 186$

\title{
Laser experiments explore the hidden sector
}

\author{
M. Ahlers ${ }^{1}$, H. Gies ${ }^{2}$, J. Jaeckel ${ }^{2,3}$, J. Redondo ${ }^{4}$ and A. Ringwald ${ }^{4}$ \\ ${ }^{1}$ Rudolf Peierls Centre for Theoretical Physics, University of Oxford, \\ 1 Keble Road, Oxford OX1 3NP, United Kingdom \\ ${ }^{2}$ Institute for Theoretical Physics, Heidelberg University, \\ Philosophenweg 16, D-69120 Heidelberg, Germany \\ ${ }^{3}$ Institute for Particle Physics Phenomenology, Durham University, \\ Durham DH1 3LE, United Kingdom \\ ${ }^{4}$ Deutsches Elektronen-Synchrotron DESY, \\ Notkestrasse 85, D-22607 Hamburg, Germany
}

\begin{abstract}
Recently, the laser experiments BMV and GammeV, searching for light shining through walls, have published data and calculated new limits on the allowed masses and couplings for axion-like particles. In this note we point out that these experiments can serve to constrain a much wider variety of hidden-sector particles such as, e.g., minicharged particles and hidden-sector photons. The new experiments improve the existing bounds from the older BFRT experiment by a factor of two. Moreover, we use the new PVLAS constraints on a possible rotation and ellipticity of light after it has passed through a strong magnetic field to constrain pure minicharged particle models. For masses $\lesssim 0.05 \mathrm{eV}$, the charge is now restricted to be less than $(3-4) \times 10^{-7}$ times the electron electric charge. This is the best laboratory bound and comparable to bounds inferred from the energy spectrum of the cosmic microwave background.
\end{abstract}




\section{Introduction}

Most extensions of the Standard Model, notably the ones based on string theory, predict a so-called "hidden sector" of particles which transform trivially under the Standard model gauge group and therefore do not directly interact via the Standard Model forces with the known particles from the "visible sector". Those hidden-sector particles typically interact via feeble, gravity-like interactions with the Standard Model. For example in supersymmetric extensions of the Standard Model, supersymmetry (SUSY) breaking is often assumed to be generated at a high scale by hidden-sector dynamics and then communicated to the minimal supersymmetric Standard Model (MSSM) sector by such forces.

Frequently, it is assumed that all the particles in the hidden sector are very heavy. This is, however, not necessarily the case. For example, the hidden sector gauge group may contain unbroken $\mathrm{U}(1)$ gauge factors, corresponding to additional massless spinone bosons, potentially mixing with the photon. Moreover, chiral symmetries may be responsible for keeping some matter particles from the hidden sector very light. Finally, if these particles are charged under the above U(1), they typically acquire a small electric charge due to the above mentioned mixing phenomenon. Thus, light minicharged particles $(\mathrm{MCPs})$ arise naturally within hidden sectors.

Laser experiments provide a powerful laboratory tool to shed light on hidden sectors with potentially tiny couplings to photons. Laser polarization experiments - such as BFRT [1], PVLAS [2], and Q\&A [3], where linearly polarized laser light is sent through a transverse magnetic field, and changes in the polarization state are searched for are sensitive to axion-like particles (ALPs) [4, 5], to MCPs [6], to hidden sector U(1) bosons [7, 8] and to other very weakly interacting sub-eV particles (WISPs) such as chameleons [9] and the like [10]. Light-shining-through-walls (LSW) experiments, such as BFRT [1, 11, are another powerful tool to search for WISPs. Here, laser light is shone onto a wall, and one searches for photons that re-appear behind the wall. Vacuum oscillations of photons into hidden-sector photons with sub-eV masses would lead to a non-vanishing regeneration rate [12]. In the presence of a magnetic field, photons can oscillate into ALPs [13, 14, 15, 16] or into massless hidden-sector photons coupling to light hidden-sector particles [7], which can be reconverted into photons behind the wall by another magnetic field.

Recently, the laser polarization experiment PVLAS [17] and the LSW experiments BMV [18] and GammeV [19] published new results. All three experiments found no significant signs of a signal and put corresponding limits on the coupling of a hypothetical ALP to a photon. But from the optical data, much more information about hidden-sector scenarios can be extracted. Further results can be expected from similar experiments such as ALPS [20], LIPSS [21], OSQAR [22], and PVLAS LSW [23] in the near future. It is the purpose of this note to explore the parameter space of hidden-sector photons and minicharged particles in the light of the new data. 


\section{$2 \quad$ Minicharged particles}

The first hypothesis which we will confront with the new data involves light particles with mass $m_{\epsilon}$ and small charges $\epsilon e$ under the electromagnetic $\mathrm{U}(1)$ : minicharged particles (MCPs). Within the context of laser experiments we expect to be sensitive only to $m_{\epsilon}$ much smaller than the electron mass where $\epsilon$ should be kept $\ll 1$. As an effective lowenergy theory, we will assume that a standard minimal coupling between the MCPs and the photon exists; moreover, we consider both fermionic Dirac spinor MCPs as well as complex scalar MCPs.

If this low-energy theory was valid even at solar energy scales $\sim \mathrm{keV}$, the astrophysics of horizontal branch stars would already imply strong constraints on the MCP parameters, resulting in $\epsilon \leq 2 \times 10^{-14}$, for $m_{\epsilon}$ below a few $\mathrm{keV}$ [24]. However, if the MCP parameters are generated by the hidden sector at scales much below $\mathcal{O}(\mathrm{keV})$, solar physics can be unaffected by these hidden-sector degrees of freedom, which instead can become visible in laboratory experiments at the sub-eV scale. A particular scenario entailing such a mechanism is the Masso-Redondo (MR) model [25], involving MCPs as well as hiddensector photons, to be discussed in Sect. 4. A model that can give rise to MCPs as low-energy degrees of freedom without requiring hidden-sector photons has been worked out in [26] within the context of warped extra dimensions.

Within the low-energy effective theory, vacuum fluctuations of MCPs induce nonlinear and nonlocal self-interactions of the electromagnetic field (cf. Fig. 1(a)). In polarization experiments where laser photons with a small amplitude propagate in a strong magnetic field, the equation of motion for the laser amplitude $a_{\mu}$ with momentum $k_{\mu}$,

$$
\left(k^{2} g_{\mu \nu}-k_{\mu} k_{\nu}+\Pi_{\mu \nu}(k \mid B)\right) a^{\nu}(k)=0
$$

involves the polarization tensor $\Pi_{\mu \nu}$ in a magnetic field $B$ [28, 29, 30]. The two transverse photon eigenmodes correspond to polarizations parallel (\| mode) and perpendicular $(\perp$ mode) to the plane spanned by the magnetic field and the propagation direction. Their dispersion relation in the form of the eigenvalues gives rise to different vacuum magnetic refractive indices $n_{\|, \perp}$ and absorption coefficients $\kappa_{\|, \perp}$ for the two modes, being related to the real and imaginary parts of the polarization tensor. The magnetized quantum vacuum is birefringent, as parameterized by $\Delta n=n_{\|}-n_{\perp}$, and exhibits dichroism, as characterized by $\Delta \kappa=\kappa_{\|}-\kappa_{\perp}$. These effects are, of course, present already in the Standard Model, predominantly owing to electron-positron fluctuations, but MCP contributions can exceed the Standard-Model effects if the MCP mass $m_{\epsilon}$ is sufficiently small [6]. The MCP-induced quantities are

$$
\Delta n=-\frac{\epsilon^{2} \alpha}{4 \pi}\left(\frac{\epsilon e B}{m_{\epsilon}^{2}}\right)^{2} \Delta I(\lambda), \quad \Delta \kappa=\frac{1}{2} \epsilon^{3} e \alpha \frac{B}{m_{\epsilon}} \Delta T(\lambda),
$$

where $\Delta I=I_{\|}-I_{\perp}($ and analogously for $\Delta T)$ and, for instance, for a Dirac spinor MCP, 


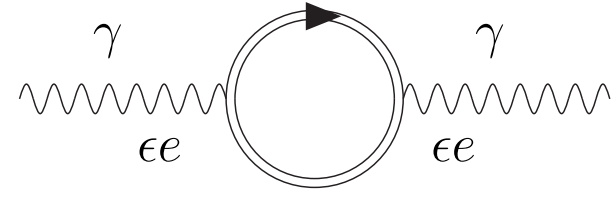

(a)

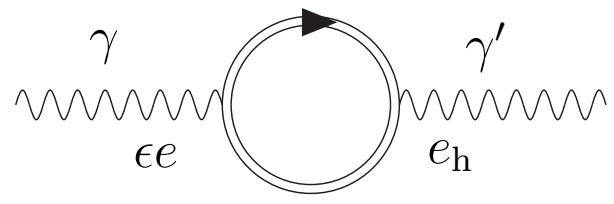

(b)

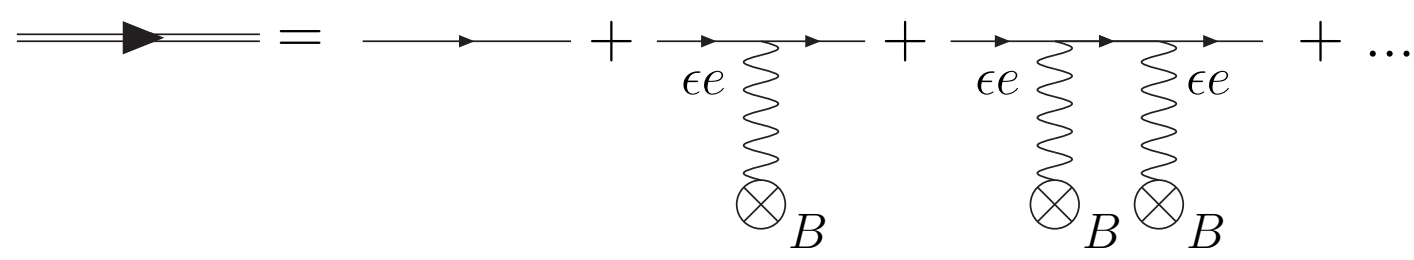

(c)

Figure 1: The contribution of minicharged particles to the polarization tensor 1(a), The real part leads to birefringence, whereas the imaginary part reflects the absorption of photons caused by the production of particle-antiparticle pairs. The analogous diagram $1(\mathrm{~b})$ shows how minicharged particles mediate transitions between photons and hidden-sector photons $\gamma^{\prime}$. Note that the latter diagram is enhanced with respect to the first one by a factor $\sim e_{\mathrm{h}} /(\epsilon e)=1 / \chi$. The double line represents the complete propagator of the minicharged particle in an external magnetic field $B$ as displayed in 1(c) [27].

we have [29]

$$
\begin{aligned}
I_{\|, \perp}^{\mathrm{Dsp}}(\lambda) & =2^{\frac{1}{3}}\left(\frac{3}{\lambda}\right)^{\frac{4}{3}} \int_{0}^{1} \mathrm{~d} v \frac{\left[\left(1-\frac{v^{2}}{3}\right)_{\|},\left(\frac{1}{2}+\frac{v^{2}}{6}\right)_{\perp}\right]}{\left(1-v^{2}\right)^{\frac{1}{3}}} \tilde{e}_{0}^{\prime}\left[-\left(\frac{6}{\lambda} \frac{1}{1-v^{2}}\right)^{\frac{2}{3}}\right], \\
T_{\|, \perp}^{\mathrm{Dsp}} & =\frac{4 \sqrt{3}}{\pi \lambda} \int_{0}^{1} \mathrm{~d} v \frac{\left[\left(1-\frac{v^{2}}{3}\right)_{\|},\left(\frac{1}{2}+\frac{v^{2}}{6}\right)_{\perp}\right]}{\left(1-v^{2}\right)} K_{2 / 3}\left(\frac{4}{\lambda} \frac{1}{1-v^{2}}\right),
\end{aligned}
$$

where $K_{\nu}(x)$ is the MacDonald function, and $\tilde{e}_{0}^{\prime}(x)$ denotes the derivative of the generalized Airy function $\tilde{e}_{0}(x)=\int_{0}^{\infty} \mathrm{d} u \sin \left(x u-u^{3} / 3\right)$. For the corresponding quantities for scalar MCPs, see the appendix of [31]. The quantity $\lambda$ abbreviates the dimensionless combination

$$
\lambda \equiv \frac{3}{2} \frac{\omega}{m_{\epsilon}} \frac{\epsilon e B}{m_{\epsilon}^{2}}
$$

where $\omega$ is the laser frequency. The above results hold for magnetic fields which are slowly varying over the scale of the MCP Compton wavelength $1 / m_{\epsilon}$. The formula for the absorption coefficient difference $\Delta \kappa$ requires the laser frequency to be above the MCP pair 

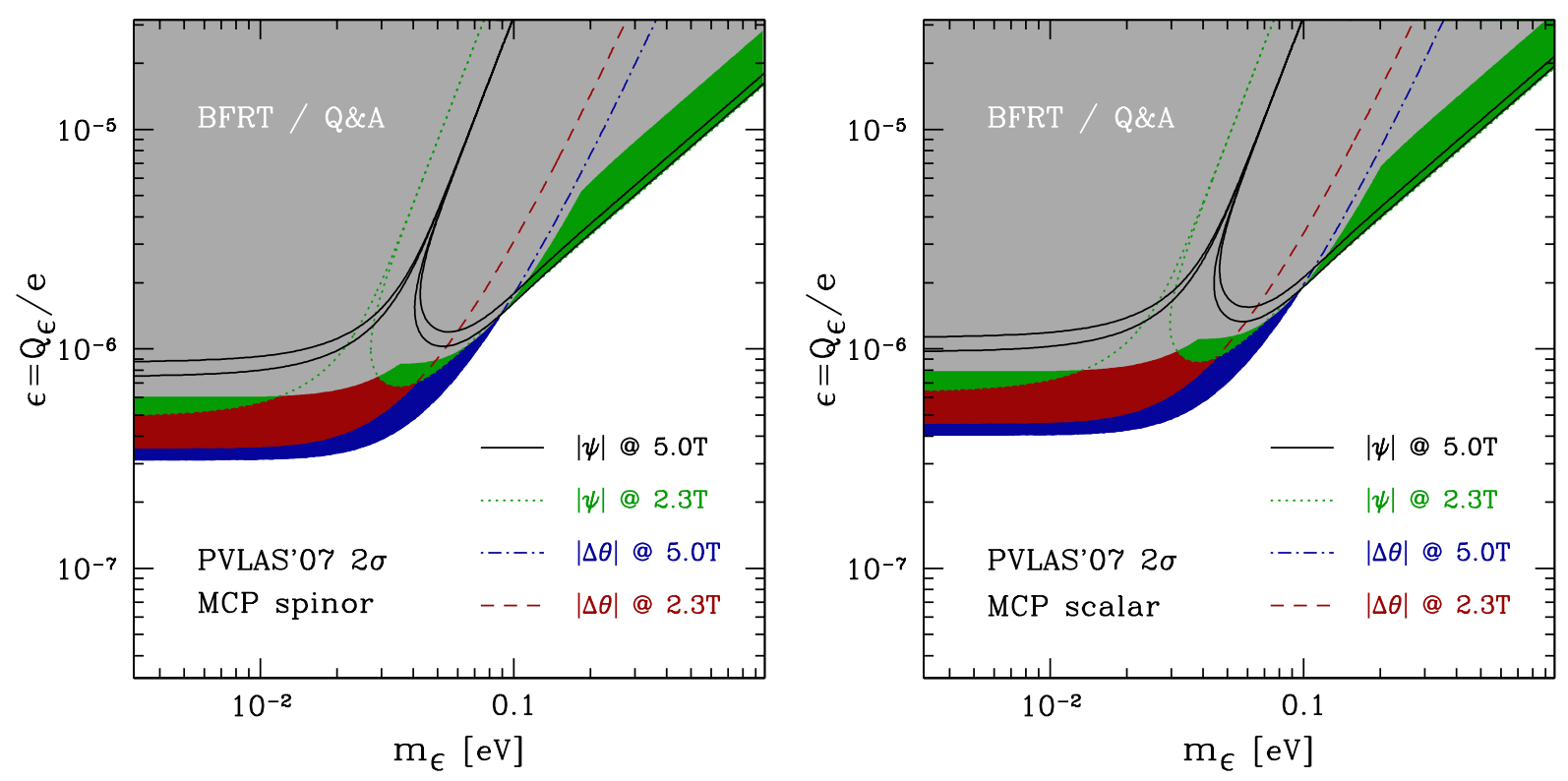

Figure 2: New constraints for the MCP mass $m_{\epsilon}$ and charge fraction $\epsilon$ for Dirac spinor MCPs (left panel) and complex scalar MCPs (right panel) as deduced from the new PVLAS data [17. Also shown are the constraints obtained from the BFRT [1] and Q\&A [3] data, as derived in Ref. [31. The constraints result from the absence of ellipticity $\psi$ and rotation $\Delta \theta$ signals, with the shaded parameter space being excluded at $95 \%$ C.L.. The $2 \sigma$ region for the ellipticity signal $\psi$ at $B=5 \mathrm{~T}$ is marked with solid lines; apart from a marginally allowed region at larger masses, this signal is excluded in the MCP scenario.

threshold $\omega \geq 2 m_{\epsilon}$, and both formulas assume that a high number of Landau levels can be occupied [30]. Given that the laser experiments we consider have $\mathcal{O}(\mathrm{m})$ lengths and $\mathcal{O}(\mathrm{eV})$ frequencies we expect our expressions to be valid roughly for $10^{-7} \mathrm{eV}<m_{\epsilon}<1 \mathrm{eV}$.

Laser polarization experiments such as BFRT, PVLAS, and Q\&A search for vacuummagnetically-induced $\Delta n$ and $\Delta \kappa$ by sending a linearly polarized laser beam containing both modes, $\|$ and $\perp$, through a strong magnetic field of $\mathcal{O}(\mathrm{T})$. If the magnetized quantum vacuum is birefringent, $\Delta n \neq 0$, the laser light picks up an ellipticity $\psi$; if it is also dichroic, $\Delta \kappa \neq 0$, the amplitudes of the two modes are depleted differently and the laser polarization undergoes an effective rotation $\Delta \theta$,

$$
\psi=\frac{\omega}{2} \ell_{B} \Delta n \sin (2 \theta), \quad \Delta \theta=\frac{1}{4} \ell_{B} \Delta \kappa \sin (2 \theta),
$$

where $\theta$ is the initial angle of polarization with respect to the direction of the $B$ field, and $\ell_{B}$ is the optical path length inside the magnetic field. Both expressions have been derived assuming $\psi, \Delta \theta \ll 1$. A summary of the experimental parameters can be found in Tabs. 1 and 2.

The PVLAS experiment 11 has recently published new constraints on $\Delta n$ and $\Delta \kappa$, re-

\footnotetext{
${ }^{1}$ In the PVLAS setup, $\theta$ is slowly changing in time covering all values from 0 to $2 \pi$. For our exclusion
} 


\begin{tabular}{lccccc}
\hline Experiment & $\omega[\mathrm{eV}]$ & $N_{\text {pass }}$ & $B_{\text {range }}[\mathrm{T}]$ & $\ell_{B}[\mathrm{~m}]$ & $|\Delta \theta|[\mathrm{nrad}]$ \\
\hline BFRT & 2.47 & 254 & $2.6-3.9$ & 8.8 & $<0.60(95 \%$ C.L. $)$ \\
Q\&A & 1.17 & 18700 & $0-2.3$ & 1.0 & $<10(95 \%$ C.L. $)$ \\
PVLAS "low field" & 2.33 & 45000 & $0-2.3$ & 1.0 & $<10(95 \%$ C.L. $)$ \\
PVLAS "high field" & 2.33 & 45000 & $0-5.0$ & 1.0 & $<12(95 \%$ C.L. $)$ \\
\hline
\end{tabular}

Table 1: Parameters of the polarization experiments searching for a possible rotation $\Delta \theta$ of the polarization after passage through a magnetic field. $\omega$ is the frequency of the laser light, $N_{\text {pass }}$ is the number of passes through the cavity of length $\ell_{B}$. $B_{\text {range }}$ denotes the range over which the magnetic field projected on a fixed direction perpendicular to the laser beam is varied. In the BFRT experiment this range is achieved by ramping the magnetic field between two different field strengths while keeping the direction of the magnetic field fixed. In the Q\&A and PVLAS experiments the magnetic field is rotated while keeping the overall field strength constant.

\begin{tabular}{lccccc}
\hline Experiment & $\omega[\mathrm{eV}]$ & $N_{\text {pass }}$ & $B_{\text {range }}[\mathrm{T}]$ & $\ell_{B}[\mathrm{~m}]$ & $|\psi|[\mathrm{nrad}]$ \\
\hline BFRT & 2.47 & 34 & $2.6-3.9$ & 8.8 & $<2.0(95 \%$ C.L. $)$ \\
PVLAS "low field" & 2.33 & 45000 & $0-2.3$ & 1.0 & $<14$ (95\% C.L.) \\
PVLAS "high field" & 2.33 & 45000 & $0-5.0$ & 1.0 & $90 \pm 9$ \\
\hline
\end{tabular}

Table 2: As in Tab. 1, but searches for a possible ellipticity of the polarization.

sulting from ellipticity $\psi$ and rotation $\Delta \theta$ measurements at $B=2.3 \mathrm{~T}$ and $B=5 \mathrm{~T}$. No signals were observed for $\Delta \theta(B=2.3 \mathrm{~T}), \Delta \theta(B=5 \mathrm{~T})$, and $\psi(B=2.3 \mathrm{~T})$; a signal was present in a $\psi(B=5 \mathrm{~T})$ measurement which is likely to result from an instrumental artifact.

These measurements translate into new bounds for the MCP parameters shown in Fig. 2 for Dirac spinors (left panel) and complex scalars (right panel). The shaded parameter regions show the excluded domain at 95\%C.L.. For instance for Dirac spinor MCPs, we find that $\epsilon \lesssim 3 \times 10^{-7}$ for $m_{\epsilon}<30 \mathrm{meV}$. This bound is indeed of a similar size as a cosmological MCP bound which has recently been derived from a conservative estimate of the distortion of the energy spectrum of the cosmic microwave background [32]. Hence, laboratory experiments begin to enter the hidden-sector parameter regime which has previously been accessible only to cosmological and astrophysical considerations.

Incidentally, the anomalous ellipticity signal $\psi(B=5 \mathrm{~T})$ (solid lines) is only marginally compatible with the other data for larger masses $m_{\epsilon} \gtrsim 0.1 \mathrm{eV}$ within the MCP hypothesis. This is in line with the PVLAS interpretation that this signal results from an instrumental artifact.

bounds we are taking just $2 \theta=\pi / 2$. 


\begin{tabular}{lcccccccccc}
\hline Experiment & $\omega[\mathrm{eV}]$ & $N_{\text {pass }}$ & $B[\mathrm{~T}]$ & $\ell_{1}[\mathrm{~m}]$ & $\ell_{2}[\mathrm{~m}]$ & $L_{1}[\mathrm{~m}]$ & $L_{2}[\mathrm{~m}]$ & $N_{0}$ & $N_{95 \%}$ \\
\hline BFRT & 2.47 & 200 & 3.7 & 4.4 & 4.4 & 11 & 6.5 & $7.8 \times 10^{18} \mathrm{~Hz}$ & 0.055 & $0.018 \mathrm{~Hz}$ \\
BMV & 1.17 & - & 12.3 & 0.365 & 0.365 & 20.0 & 1.0 & $6.7 \times 10^{22}$ & 0.5 & 3.09 \\
GammeV "centre" & 2.33 & - & 5.0 & 3.1 & 2.9 & 5.4 & 7.2 & $6.6 \times 10^{23}$ & 0.33 & 3.69 \\
GammeV "edge" & 2.33 & - & 5.0 & 5.0 & 1.0 & 7.3 & 7.1 & $6.4 \times 10^{23}$ & 0.33 & 2.05 \\
\hline
\end{tabular}

Table 3: Parameters of LSW experiments. Here $\omega$ is the frequency of the laser beam, $N_{\text {pass }}$ the number of passes through the cavity (if present) and $B$ the magnetic field strength. $\ell_{1}$ and $\ell_{2}$ are the lengths of the magnetized regions of the production and regeneration sides of the experiments whereas $L_{1}$ and $L_{2}$ are the total lengths on both sides including regions without magnetic field. $N_{0}$ is the photon number or rate of the laser beam, $\eta$ the quantum efficiency of the detector and $N_{95 \%}$ the upper limit on the detected number of photons. We follow Ref. 43 . in deriving the $95 \%$ C.L. upper limits $\left(N_{95 \%}\right)$ for the BMV and GammeV results.

\section{Massive hidden-sector photons}

The next hypothesis which we will confront with data is based on the assumption that the low-energy dynamics involves, in addition to the familiar massless electromagnetic $\mathrm{U}(1)_{\mathrm{QED}}$, another hidden-sector $\mathrm{U}(1)_{\mathrm{h}}$ under which all Standard Model particles have zero charge. The most general renormalizable Lagrangian describing these two U(1) gauge groups at low energies is

$$
\mathcal{L}=-\frac{1}{4} F^{\mu \nu} F_{\mu \nu}-\frac{1}{4} B^{\mu \nu} B_{\mu \nu}-\frac{1}{2} \chi F^{\mu \nu} B_{\mu \nu}+\frac{1}{2} m_{\gamma^{\prime}}^{2} B_{\mu} B^{\mu}
$$

where $F_{\mu \nu}$ is the field strength tensor for the ordinary electromagnetic $\mathrm{U}(1)_{\mathrm{QED}}$ gauge field $A^{\mu}$, and $B^{\mu \nu}$ is the field strength for the hidden-sector $\mathrm{U}(1)_{\mathrm{h}}$ field $B^{\mu}$. The first two terms are the standard kinetic terms for the photon and hidden-sector photon fields, respectively. Because the field strength itself is gauge invariant for $\mathrm{U}(1)$ gauge fields, the third term is also allowed by gauge and Lorentz symmetry. This term corresponds to a non-diagonal kinetic term, a so-called kinetic mixing [33, 34]. From the viewpoint of a low-energy effective Lagrangian, $\chi$ is a completely arbitrary parameter. Embedding the model into a more fundamental theory, it is plausible that $\chi=0$ holds at a high-energy scale related to the fundamental theory. However, integrating out the heavy quantum fluctuations generally tends to generate non-vanishing $\chi$ at low scales. Indeed, kinetic mixing arises quite generally both in field theoretic [33, 35] as well as in string theoretic [36, 37, 38, 39, 40, 41] setups, and typical predicted values for $\chi$ range between $10^{-16}$ and $10^{-4}$. The last term in the Lagrangian (3.1) accounts for a possible mass of the paraphoton. This may arise via a Higgs or, alternatively, via a Stueckelberg [42] mechanism.

Let us now switch to a field basis in which the prediction of photon $\leftrightarrow$ hidden-sector photon oscillations becomes apparent. In fact, the kinetic terms in the Lagrangian (3.1) can be diagonalized by a shift

$$
B^{\mu} \rightarrow \tilde{B}^{\mu}-\chi A^{\mu}
$$


Apart from a multiplicative renormalization of the electromagnetic gauge coupling, $e^{2} \rightarrow$ $e^{2} /\left(1-\chi^{2}\right)$, the visible-sector fields remain unaffected by this shift and one obtains a non-diagonal mass term that mixes photons with hidden-sector photons,

$$
\mathcal{L}=-\frac{1}{4} F^{\mu \nu} F_{\mu \nu}-\frac{1}{4} \tilde{B}^{\mu \nu} \tilde{B}_{\mu \nu}+\frac{1}{2} m_{\gamma^{\prime}}^{2}\left(\tilde{B}^{\mu} \tilde{B}_{\mu}-2 \chi \tilde{B}^{\mu} A_{\mu}+\chi^{2} A^{\mu} A_{\mu}\right)
$$

where we have absorbed an irrelevant factor of $\sqrt{1-\chi^{2}}$ in both $A_{\mu}$ and $1 / \chi$. Therefore, in analogy to neutrino flavour oscillations, photons may oscillate in vacuum into hiddensector photons. These oscillations and the fact that hidden-sector photons do not interact with ordinary matter forms the basis of the possibility to search for signals of hiddensector photons in LSW experiments. The probability for a photon to pass through the wall and to arrive at the detector is [12, 7],

$$
P_{\text {trans }}=16 \chi^{4}\left[\sin \left(\frac{\Delta k L_{1}}{2}\right) \sin \left(\frac{\Delta k L_{2}}{2}\right)\right]^{2},
$$

where $L_{1}\left(L_{2}\right)$ is the distance between the laser and the wall (the wall and the detector), and

$$
\Delta k=\omega-\sqrt{\omega^{2}-m_{\gamma^{\prime}}^{2}} \approx m_{\gamma^{\prime}}^{2} /(2 \omega), \quad \text { for } m_{\gamma^{\prime}} \ll \omega,
$$

is the momentum difference between the photon and the hidden-sector photon, expressed in terms of the energy of the laser photons, $\omega$. If a cavity is used on the production side, i.e., before the wall, Eq. (3.4) receives an additional factor of $\left(N_{\text {pass }}+1\right) / 2$, where $N_{\text {pass }} \gg 1$ is the number of passes the light makes through the cavity; also, the length $L_{1}$ then has to be replaced by the path length $\ell_{1}$ inside the cavity. The LSW transition probability in Eq. (3.4) is actually independent of the magnetic field, since the mixing arises from the mass term. The expected rate of observed photons in addition involves the total initial photon rate $N_{0}$ and the detection efficiency $\eta<1, N=\eta N_{0} P_{\text {trans }}$. The experimental parameters of BFRT [1], BMV [18] and GammeV [19], as relevant for the search to paraphotons, are summarized in Table 3 .

Using the constraints on the number of photons passing through the wall obtained from the experiments of Tab. 3 we can obtain new bounds in the mass-mixing plane as shown in the left panel in Fig. 3, Combining the results of the new experiments results in an improvement by roughly a factor of two compared to the older bounds from BFRT over a wide range of masses. In the mass range $10^{-4} \mathrm{eV} \lesssim \mathrm{m}_{\gamma^{\prime}} \lesssim 10^{-2} \mathrm{eV}$, these bounds are the best existing bounds on the kinetic mixing of hidden-sector photons. (cf. right panel in Fig. 3).

\footnotetext{
${ }^{2}$ More precisely, it is the length between the last optical device that redirects the laser beam light into the wall and the wall itself. See Appendix B of Ref. [7].
} 

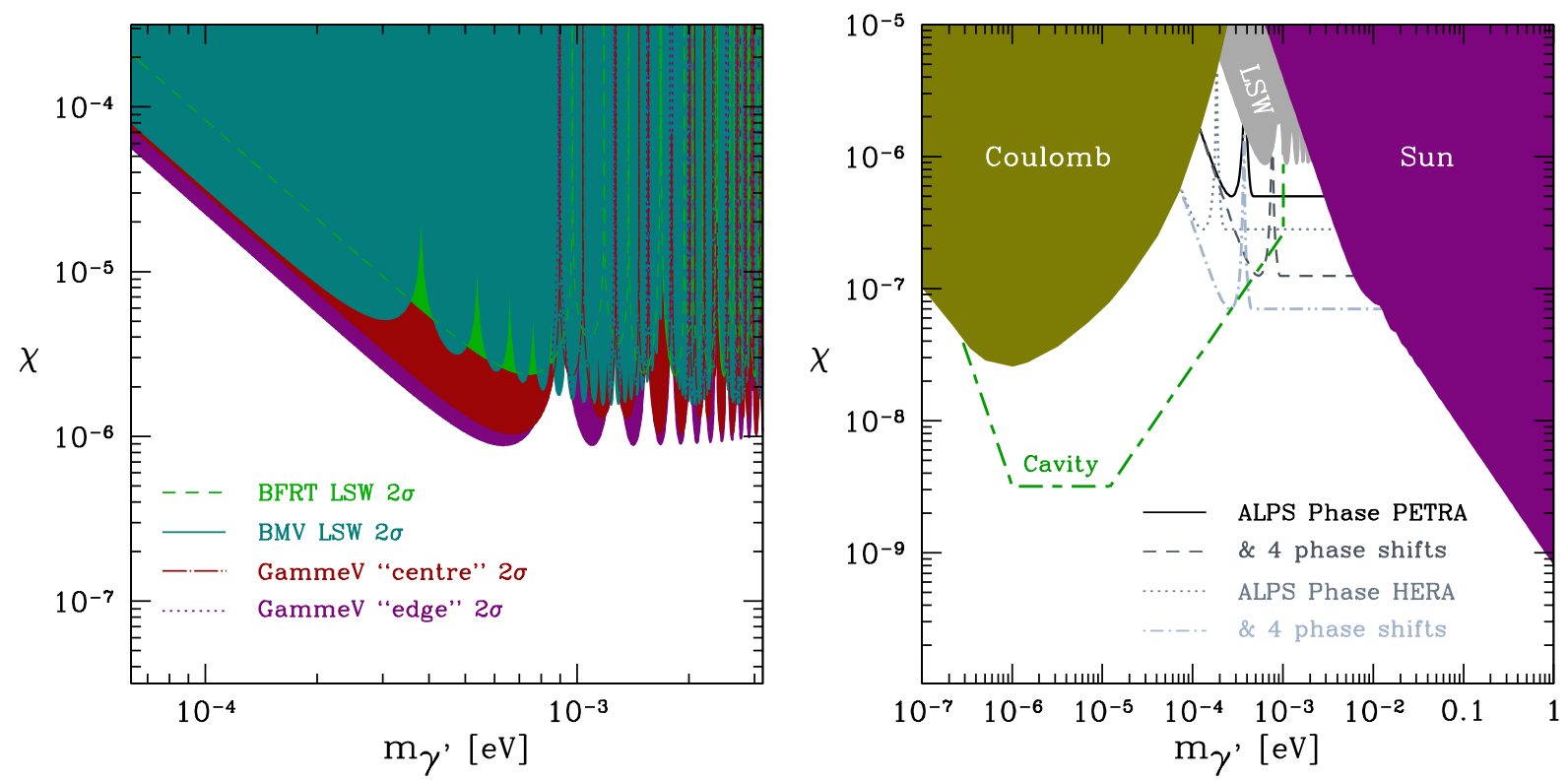

Figure 3: Limits on hidden-sector photons $\gamma^{\prime}$ mixing with the photon from searches for photon regeneration in LSW experiments. Left panel: New limits from the non-observation in the LSW experiments BMV and GammeV compared to the old BFRT results. The bounds relax by a factor $1 \sim 3$ in the MR model [25] depending on $m_{\epsilon}$. See Sect. 4 for details. Right panel: Forecast of future experiments searching for $\gamma^{\prime}$ s. The current limit from LSW experiments (grey shaded) can be extended by dedicated experiments exploiting high power, $\sim 200 \mathrm{~W}$, lasers with long beamlines, e.g. $L_{1}=L_{2}=40 \mathrm{~m}$ for ALPS [20] Phase PETRA (black solid) or $L_{1}=L_{2}=170 \mathrm{~m}$ for ALPS Phase HERA (blue dotted). Inserting "phase-shift plates" into the beamline as suggested in Ref. [44] could improve the LSW results at larger masses. A substantial improvement in the sensitivity to $\chi$ by several orders of magnitude can be achieved, in the mass range from $m_{\gamma^{\prime}} \sim 10^{-7} \mathrm{eV}$ to $m_{\gamma^{\prime}} \sim 10^{-4} \mathrm{eV}$, through experiments exploiting highquality microwave cavities [45]. These experiments are complementary to searches for deviations of the Coulomb law [46, 47] and for photon regeneration of hidden-sector photons produced in the Sun within the CAST magnet [48] (the limit arising from the lifetime of the Sun is slightly worse (see also Ref. [49])).

\section{Hidden-sector photons and minicharged particles}

In Sect. 2 we have simply assumed the existence of light minicharged particles without any additional light particles being present. However, in many models the minicharges actually arise from the coupling of a hidden-sector particle to a hidden-sector photon that has a kinetic mixing with the ordinary photon [33]. In other words we have minicharged particles and hidden-sector photons.

Let us briefly recall how minicharged particles arise from kinetic mixing. Assume that we have a hidden sector fermion ${ }^{3} h$ that has charge one under $B^{\mu}$. Applying the shift (3.2)

\footnotetext{
${ }^{3}$ Here, and in the following we will specialize to the case where the hidden sector particle is a fermion.
} 
to the coupling term, we find:

$$
e_{h} \bar{h} \not B h \rightarrow e_{h} \bar{h} \tilde{B} h-\chi e_{h} \bar{h} A h,
$$

where $e_{h}$ is the hidden sector gauge coupling. We can read off that the hidden sector particle now has a charge

$$
\epsilon e=-\chi e_{h}
$$

under the visible electromagnetic gauge field $A^{\mu}$ which has gauge coupling $e$. For small $\chi \ll 1$, we notice that

$$
|\epsilon| \ll 1
$$

and $h$ becomes a minicharged particle.

However, from Eq. (3.3) we can see that for $m_{\gamma^{\prime}} \neq 0$ the photon propagator has off-diagonal elements. One finds that for momenta $q \ll m_{\gamma^{\prime}}$ these off-diagonal elements cancel the effect of the minicharge (for details see, e.g., Ref. [7]) and the coupling to $h$ is effectively zerd 4 . In most laboratory experiments the typical momenta are often tiny. Therefore, if we want to have additional effects from minicharged particles the most interesting case is that of a massless (or nearly massless) hidden-sector photon.

If the hidden-sector photon is massless, the photon $\leftrightarrow$ hidden-sector photon oscillations cannot take place via a mass term as in Sect. 3. However, due to presence of the additional (light) minicharged fermions it can take place via a loop diagram as shown in Fig. 1(b), This process is now possible in addition to the production of minicharged particles as discussed in Sect. 2 that leads to an imaginary part of the photon polarization tensor (Fig. 1(a)]. The transition probability after a distance $z$ is [7],

$$
P_{\gamma \rightarrow \gamma^{\prime}}^{i}(z)=P_{\gamma^{\prime} \rightarrow \gamma}^{i}(z)=\chi^{2}\left[1+\exp \left(-\kappa_{i} z / \chi^{2}\right)-2 \exp \left(-\kappa_{i} z /\left(2 \chi^{2}\right)\right) \cos \left(\Delta n_{i} \omega z / \chi^{2}\right)\right],
$$

where $i=\|, \perp$ denotes the polarization parallel or perpendicular to the magnetic field. The total light-shining-through-a-wall probability is then,

$$
P_{\text {trans }}=\left[\frac{N_{\text {pass }}+1}{2}\right] P_{\gamma \rightarrow \gamma^{\prime}}\left(\ell_{1}\right) P_{\gamma^{\prime} \rightarrow \gamma}\left(\ell_{2}\right),
$$

where $\ell_{1}\left(\ell_{2}\right)$ denotes the length of the magnetic field in front (behind) the wall.

Using the new experimental bounds, Eq. (4.5) can be turned into a bound on the possible amount of kinetic mixing in a model with one massless hidden-sector photon, which is shown in the left panel of Fig. 4 (see Tab. 3 for experimental parameters). The new experiments improve the existing bounds from BFRT roughly by a factor of two. Also, for completeness we show in the right panel the dependence of these bounds on the a priori unknown hidden sector gauge coupling $e_{h}$ in the particularly simple, yet enlightening, case of a massless MCP.

A generalization to scalars is straightforward and does not change the results qualitatively.

${ }^{4}$ To be exact, this is true only if the physical size of the magnetic field region is larger than the inverse hidden-secton photon mass. This is always the case for the parameter regions which we are exploring in this note. For further details see [7]. 

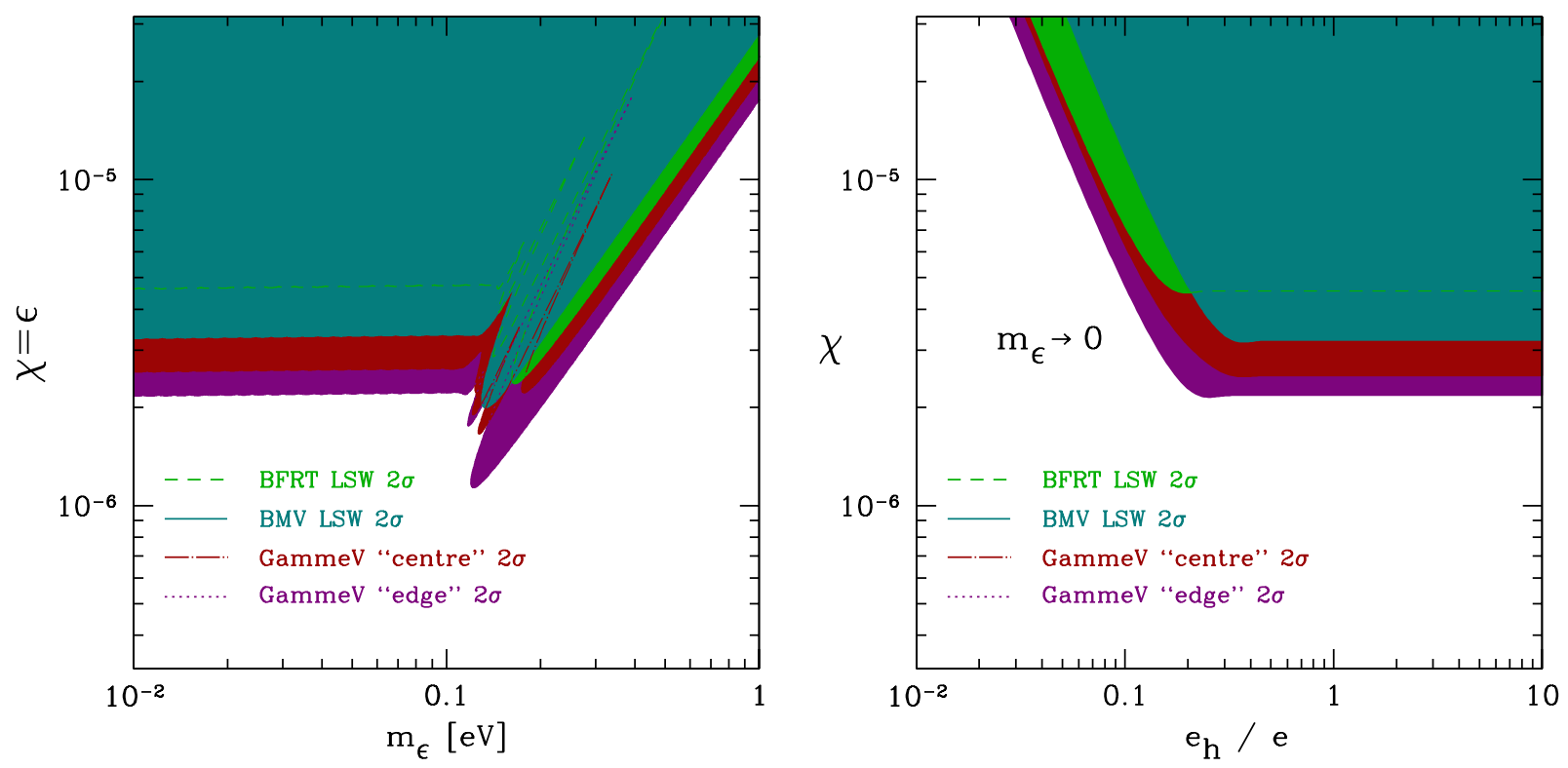

Figure 4: Left panel: Limits on the kinetic mixing of hidden-sector photons with the ordinary electromagnetic photon in a model with a minicharged particle with charge $\epsilon=\chi$, i.e. $e_{h}=-e$. Right panel: Limits on the kinetic mixing parameter $\chi$ as a function of the hidden sector gauge coupling $e_{h}$ in a model with a minicharged particle with $m_{\epsilon} \rightarrow 0$.

Finally we would like to comment on the implications of the discussed experiments for the MR model [25], still one of the few possibilities to evade the strong astrophysical bounds for light MCPs. In its minimal version 5 [40, 7], it features three new particles: two hidden-sector photons, $B_{ \pm}$, the first one mixing kinetically with the ordinary photon with mixing parameter 6 and the second just coupling minimally to a hidden sector fermion $h$. If the $B_{ \pm}$photons were massless, the model would not have any phenomenological consequences. However, adding a non-diagonal mass term $\mu^{2}\left(B_{+}+B_{-}\right)^{2} / 4$ in the Lagrangian leads to a "link" between the photon and $h$. This link is realized as a $\mu^{2}$ dependent minicharge that relaxes to zero if the mass vanishes or if it is much smaller than the other energy scales involved in the particular process under consideration. This latter case includes the production of $h$ particles in cosmological or astrophysical environments where the energy can be much larger than $\mu$, for $\mu \lesssim \mathrm{eV}$. In Ref. [25] it is claimed that models satisfying $7 \mu^{2} \lesssim 6 \times 10^{-9} \mathrm{eV}^{2}$ do not suffer from excessive anomalous energy loss in horizontal branch stars of globular clusters, the most important constraint for the existence of sub-eV MCPs (see however [32]).

All these particles can show up in laser experiments as long as $\mu$ is not excessively small. Let us focus on a LSW type of experiment 8 Generically, their signature is similar

\footnotetext{
${ }^{5}$ In Ref. [25], the authors include also an additional spin-zero particle.

${ }^{6}$ The value of this mixing would be $\sqrt{2} \chi$ in 25 . We have redefined it for practical purposes.

${ }^{7}$ The authors consider $\left|e_{h}\right|=|e|$.

${ }^{8}$ One finds (see Ref. [7]) that rotation and ellipticity experiments are sensitive for a smaller part of the parameter space $\left(\chi, \mu, m_{\epsilon}\right)$.
} 
to the signature of a hidden-photon model in such a way that we can use Fig. 3 with $m_{\gamma^{\prime}}=\mu$ as a rough estimate of the exclusion bounds on the MR model as well. In fact, the MR model reduces to the hidden photon situation developed in Sec. 3 for $m_{\epsilon} \rightarrow \infty$. Finally, we have checked numerically that for the opposite limiting case, namely $m_{\epsilon}=0$, the combined bounds relax by no more than a factor three in the region of Fig. 3 (left panel). We believe that for finite values of $m_{\epsilon}$ the combined bounds should roughly lie between these two limiting cases. A detailed study of the MR model parameter space is, however, beyond the scope of this note.

\section{Conclusions}

Axion-like particles are the "usual suspects" investigated in laser polarization and lightshining-through-a-wall (LSW) experiments. However, the non-observation of a significant signal so far constrains also alternative scenarios, like hidden-sector photons and minicharged particles.

We have shown that the recent PVLAS limits on the birefringence and dichroism of a magnetized vacuum constrain the charge of minicharged particles with masses $\lesssim 0.05 \mathrm{eV}$ to be less than $(3-4) \times 10^{-7}$ times the electron electric charge. This is the best laboratory bound and comparable to bounds from the cosmic microwave background although it is still far from the astrophysical limits. The latter are, however, associated with physics at a much higher energy scale and their application to the low energy physics probed by laboratory experiments requires an extrapolation over many orders of magnitude.

Moreover, the LSW limits of BMV and GammeV improve the existing bounds from BFRT on the coupling and mixing of hidden-sector photons. In the case of massive paraphotons in the mass range $10^{-4} \mathrm{eV} \lesssim m_{\gamma^{\prime}} \lesssim 10^{-2} \mathrm{eV}$, the bound is improved by about a factor two. These are currently the best existing bounds on hidden-sector photons around $m_{\gamma^{\prime}} \lesssim 10^{-3} \mathrm{eV}$ and they could be improved even by one order of magnitude in the near future.

Similarly, for models where minicharged particles acquire their charge through kinetic mixing with massless hidden-sector photons, the LSW bounds on the kinetic mixing parameter are improved by about the same factor two.

From a general viewpoint, laser experiments have demonstrated their capability of exploring new domains in the particle-physics parameter space, being particularly powerful for weakly coupled light particles. In addition to typical hidden-sector degrees of freedom, also new fields arising in the context of cosmological models could be searched for by similar optical techniques; see, e.g., [50, 51]. In the light of hidden-sector physics, it is worthwhile to reconsider also other concepts on using optical or more general electromagnetic signatures to deduce information about the underlying particle-physics content, 
e.g., using strong laser fields [52, 53, 54], strong electric fields inside cavities [55], or astronomical observations [56, 57, 58].

\section{Acknowledgements}

We thank Carlo Rizzo, Giuseppe Ruoso, and William Wester for kindly providing us details of their light-shining-through-walls experiments. MA acknowledges support by STFC UK (PP/D00036X/1). HG acknowledges support by the DFG under contract No. Gi 328/1-4 (Emmy-Noether program).

\section{References}

[1] R. Cameron et al. [BFRT Collaboration], Phys. Rev. D 47 (1993) 3707.

[2] E. Zavattini et al. [PVLAS Collaboration], Phys. Rev. Lett. 96 (2006) 110406 arXiv:hep-ex/0507107.

[3] S. J. Chen, H. H. Mei and W. T. Ni [Q\&A Collaboration], arXiv:hep-ex/0611050.

[4] L. Maiani, R. Petronzio and E. Zavattini, Phys. Lett. B 175 (1986) 359.

[5] G. Raffelt and L. Stodolsky, Phys. Rev. D 37 (1988) 1237.

[6] H. Gies, J. Jaeckel and A. Ringwald, Phys. Rev. Lett. 97 (2006) 140402 arXiv:hep-ph/0607118.

[7] M. Ahlers, H. Gies, J. Jaeckel, J. Redondo and A. Ringwald, arXiv:0706.2836 [hepph].

[8] I. Antoniadis, A. Boyarsky and O. Ruchayskiy, arXiv:0708.3001 [hep-ph].

[9] P. Brax, C. van de Bruck, A. C. Davis, D. F. Mota and D. J. Shaw, Phys. Rev. D 76 (2007) 085010 [arXiv:0707.2801 [hep-ph]].

[10] J. Jaeckel, E. Masso, J. Redondo, A. Ringwald and F. Takahashi, Phys. Rev. D 75 (2007) 013004 arXiv:hep-ph/0610203.

[11] G. Ruoso et al. [BFRT Collaboration], Z. Phys. C 56 (1992) 505.

[12] L. B. Okun, Sov. Phys. JETP 56 (1982) 502 [Zh. Eksp. Teor. Fiz. 83 (1982) 892].

[13] P. Sikivie, Phys. Rev. Lett. 51 (1983) 1415 [Erratum-ibid. 52 (1984) 695].

[14] A. A. Anselm, Yad. Fiz. 42 (1985) 1480. 
[15] M. Gasperini, Phys. Rev. Lett. 59 (1987) 396.

[16] K. Van Bibber, N. R. Dagdeviren, S. E. Koonin, A. Kerman, and H. N. Nelson, Phys. Rev. Lett. 59 (1987) 759.

[17] E. Zavattini et al. [PVLAS Collaboration], arXiv:0706.3419 [hep-ex].

[18] C. Robilliard, R. Battesti, M. Fouche, J. Mauchain, A. M. Sautivet, F. Amiranoff and C. Rizzo [BMV Collaboration], Phys. Rev. Lett. 99 (2007) 190403 [arXiv:0707.1296 [hep-ex]].

[19] A. S. Chou et al. [GammeV (T-969) Collaboration], arXiv:0710.3783 [hep-ex].

[20] K. Ehret et al. [ALPS Collaboration], arXiv:hep-ex/0702023; see also http://alps.desy.de

[21] A. V. Afanasev, O. K. Baker and K. W. McFarlane, arXiv:hep-ph/0605250.

[22] P. Pugnat et al. [OSQAR Collaboration], CERN-SPSC-2006-035; see http://graybook.cern.ch/programmes/experiments/OSQAR.html

[23] G. Cantatore for the [PLVAS Collaboration], talk at the 3rd Joint ILIAS-CERNDESY Axion-WIMPs Training Workshop, 19-25 June 2007, University of Patras, Greece; http://axion-wimp.desy.de

[24] S. Davidson, S. Hannestad and G. Raffelt, JHEP 0005 (2000) 003 arXiv:hep-ph/0001179.

[25] E. Masso and J. Redondo, Phys. Rev. Lett. 97 (2006) 151802 |arXiv:hep-ph/0606163|.

[26] B. Batell and T. Gherghetta, Phys. Rev. D 73 (2006) 045016 arXiv:hep-ph/0512356].

[27] J. S. Schwinger, Phys. Rev. 82 (1951) 664.

[28] J.S. Toll, The Dispersion relation for light and its application to problems involving electron pairs, PhD thesis, RX-1535, Princeton University, 1952 (unpublished); V. Baier and V. Katkov, Zh. Eksp. Teor. Fiz. 53 (1967) 1478; S.L. Adler, Annals Phys. 67 (1971) 599; C. Schubert, Nucl. Phys. B 585 (2000) 407.

[29] W.-y. Tsai and T. Erber, Phys. Rev. D10 (1974) 492; Phys. Rev. D12 (1975) 1132; W. Dittrich and H. Gies, Springer Tracts Mod. Phys. 166 (2000) 1.

[30] J.K. Daugherty and A.K. Harding, Astrophys. J. 273 (1983) 761.

[31] M. Ahlers, H. Gies, J. Jaeckel and A. Ringwald, Phys. Rev. D 75 (2007) 035011 arXiv:hep-ph/0612098.

[32] A. Melchiorri, A. Polosa and A. Strumia, Phys. Lett. B 650 (2007) 416 arXiv:hep-ph/0703144. 
[33] B. Holdom, Phys. Lett. B 166 (1986) 196.

[34] R. Foot and X. G. He, Phys. Lett. B 267 (1991) 509.

[35] R. Foot and A. Kobakhidze, Phys. Lett. B 650 (2007) 46 arXiv:hep-ph/0702125.

[36] K. R. Dienes, C. F. Kolda and J. March-Russell, Nucl. Phys. B 492 (1997) 104 hep-ph/9610479.

[37] D. Lüst and S. Stieberger, Fortsch. Phys. 55 (2007) 427 arXiv:hep-th/0302221].

[38] S. A. Abel and B. W. Schofield, Nucl. Phys. B 685 (2004) 150 hep-th/0311051.

[39] R. Blumenhagen, S. Moster and T. Weigand, Nucl. Phys. B 751 (2006) 186 hep-th/0603015.

[40] S. A. Abel, J. Jaeckel, V. V. Khoze and A. Ringwald, hep-ph/0608248.

[41] J. E. Kim, Phys. Rev. D 76 (2007) 051701 arXiv:0704.3310 [hep-ph]].

[42] E. C. G. Stückelberg, Helv. Phys. Acta 11 (1938) 225.

[43] G. J. Feldman and R. D. Cousins, Phys. Rev. D 57 (1998) 3873 arXiv:physics/9711021.

[44] J. Jaeckel and A. Ringwald, Phys. Lett. B 653 (2007) 167 arXiv:0706.0693 [hep-ph]].

[45] J. Jaeckel and A. Ringwald, arXiv:0707.2063 [hep-ph].

[46] E. R. Williams, J. E. Faller and H. A. Hill, Phys. Rev. Lett. 26 (1971) 721.

[47] D. F. Bartlett and S. Loegl, Phys. Rev. Lett. 61 (1988) 2285.

[48] J. Redondo, DESY 07-211.

[49] V. V. Popov and O. V. Vasil'ev, Europhys. Lett. 15 (1991) 7; Turk. J. Phys. 23 (1999) 943.

[50] M. Ahlers, A. Lindner, A. Ringwald, L. Schrempp and C. Weniger, arXiv:0710.1555 [hep-ph].

[51] H. Gies, D. F. Mota and D. J. Shaw, arXiv:0710.1556 [hep-ph].

[52] T. Heinzl, B. Liesfeld, K. U. Amthor, H. Schwoerer, R. Sauerbrey and A. Wipf, Opt. Commun. 267 (2006) 318 arXiv:hep-ph/0601076.

[53] A. Di Piazza, K. Z. Hatsagortsyan and C. H. Keitel, Phys. Rev. Lett. 97 (2006) 083603 arXiv:hep-ph/0602039.

[54] M. Marklund and P. K. Shukla, Rev. Mod. Phys. 78, 591 (2006) arXiv:hep-ph/0602123. 
[55] H. Gies, J. Jaeckel and A. Ringwald, Europhys. Lett. 76 (2006) 794 arXiv:hep-ph/0608238.

[56] A. Dupays, C. Rizzo, M. Roncadelli and G. F. Bignami, Phys. Rev. Lett. 95 (2005) 211302 arXiv:astro-ph/0510324.

[57] A. Mirizzi, G. G. Raffelt and P. D. Serpico, arXiv:0704.3044 [astro-ph].

[58] A. De Angelis, O. Mansutti and M. Roncadelli, arXiv:0707.2695 [astro-ph]. 